\title{
PESO MÁXIMO DA MOCHILA RECOMENDADO PARA CRIANÇAS EM CONTEXTO ESCOLAR: UMA SCOPING REVIEW
}

PESO MÁXIMO RECOMENDADO DE LA MOCHILA PARA NIÑOS EN EL CONTEXTO ESCOLAR: UNA REVISIÓN DEL ALCANCE

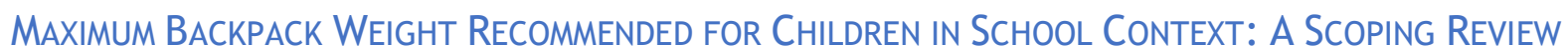

DOI 10.33194/rper.2020.v3.n1.6.5759 | Submetido 12/02/2020 | Aprovado 12/08/2020

\author{
Maria Matos $^{2}$; Catarina Barreiras ${ }^{1}$; Constança Festas $^{2}$ (i) \\ 1 - Unidade Local de Saúde do Alto Minho; \\ 2 - Universidade Católica Portuguesa, Centro de Investigação Interdisciplinar em Saúde, Instituto de Ciências da saúde
}

\section{RESUMO}

Objetivos: mapear a evidência em relação ao peso máximo da mochila recomendado para crianças em contexto escolar.

Metodologia: sugerida pelo Joanna Briggs Institute. A análise de relevância dos artigos, a extração e síntese dos dados desenvolveu-se por dois revisores independentes. Questão de partida: qual o peso máximo da mochila recomendado para crianças, dos 6 aos 12 anos, em contexto escolar? Fontes de dados: estudos primários publicados em bases de dados científicas, diretrizes internacionais e literatura cinzenta.

Síntese dos dados: identificaram-se 353 publicações, onde foram incluídas 28. A percentagem de peso recomendada subdivide-se em duas categorias: com recomendação (que oscila entre 5\%-20\%) e sem recomendação.

Conclusões: Esta scoping review permitiu mapear a evidência em relação ao peso máximo da mochila recomendado para crianças em contexto escolar, onde o valor de $10 \%$ foi o que obteve maior consenso.

Descritores: Criança, Suporte de carga, Escolas, Serviços de saúde escolar, Enfermagem em Reabilitação

\section{RESUMEN}

Objetivos: mapear la evidencia en relación con el peso máximo de mochila recomendado para niños en el contexto escolar.

Metodología: sugerida por el Instituto Joanna Briggs. El análisis de relevancia de los artículos, la extracción y síntesis de los datos fue realizado por dos revisores independientes. Pregunta inicial: ¿cuál es el peso máximo de la mochila recomendado para niños de 6 a 12 años en un contexto escolar? Fuentes de datos: estudios primarios publicados en bases de datos científicas, directrices internacionales y literatura gris.

Resumen de los datos: se identificaron 353 publicaciones, de las cuales se incluyeron 28. El porcentaje en peso recomendado se divide en dos categorías: con recomendación (que va del $5 \%$ al 20\%) y sin recomendación.

Conclusiones: Esta revisión de alcance permitió mapear la evidencia en relación con el peso máximo de la mochila recomendado para niños en la escuela, donde el valor del 10\% fue el que obtuvo el mayor consenso.

Palavras clave: Niño, Carga de peso, Escuelas, Servicios de salud escolar, Enfermería en Rehabilitación

\section{ABSTRACT}

Objectives: to map the evidence in relation to the maximum backpack weight recommended for children in school context.

Methodology: suggested by the Joanna Briggs Institute. The relevance analysis of the articles, the extraction and synthesis of the data was carried out by two independent reviewers. Starting question: what is the maximum backpack weight recommended for children, from 6 to 12 years old, in school context Data sources: primary studies published in scientific databases, international guidelines and gray literature.

Summary of the data: 353 publications were identified, of which 28 were included. The recommended weight percentage is divided into two categories: with recommendation (ranging from 5\%-20\%) and without recommendation.

Conclusions: This scoping review allowed to map the evidence in relation to the maximum weight of the backpack recommended for children in school, where the value of $10 \%$ was the one that obtained the greatest consensus.

Keywords: Child, Weight-Bearing, Schools, School Health Services, Rehabilitation Nursing 


\section{INTRODUÇÃO}

A utilização regular de mochilas escolares, frequentemente pesadas e/ou desajustadas, que comportam os manuais e materiais para todo o dia escolar, apresenta uma multiplicidade de riscos, sobretudo durante o período de crescimento de uma criança ${ }^{(1-3)}$.

De facto, as crianças e os jovens, ainda em processo de crescimento, possuem uma maior vulnerabilidade e imaturidade do sistema musculoesquelético, pois a ossificação está incompleta e existe uma grande quantidade de tecido cartilaginoso, que contribui para um aumento da incidência de lesões musculoesqueléticas (LME) por sobrecarga ${ }^{(4)}$, nomeadamente a escoliose, a hipercifose e a hiperlordose em várias fases do crescimento ${ }^{(5-6)}$.

De acordo com o Programa Nacional de Saúde Escolar (2015-2020), as LME nos estudantes resultam, frequentemente, da sobrecarga física associada ao peso em excesso de mochilas, à adoção de posturas incorretas, por inadaptação do mobiliário escolar às suas características antropométricas e à atividade desportiva inadequada, quer pelo tipo de prática ou pelo excesso de exercício ${ }^{(7)}$.

Desde 1977 que há referência científica em relação à percentagem de peso que uma criança deverá transportar na mochila escolar, a qual não deverá exceder os $10 \%$ do peso corporal da mesma, ou seja, o limite máximo aceitável será de $1 / 8$ do peso corporal ${ }^{(8)}$.

Desde então, este valor tem sido aceite pela comunidade científica ${ }^{(3,9)}$, havendo, contudo, estudos que contrapõe esta percentagem, sugerindo outros valores ${ }^{(10-11)}$, ou mesmo referindo que não é apropriado sugerir para todas as crianças o mesmo limite de peso da mochila, ou seja, um único limite de peso pode não ser adequado para todos os alunos ${ }^{(12)}$.

Depreende-se, assim, que a percentagem de peso da mochila escolar recomendada ainda não é unânime, sendo que esta problemática é envolta de uma complexidade multifatorial que deve merecer toda a atenção da comunidade escolar (CE), que inclui estudantes, professores, pais e outros profissionais( ${ }^{(7)}$, da saúde escolar e comunidade científica, através de uma revisão de literatura mais aprofundada, pela metodologia scoping review, uma vez que após a realização de uma pesquisa preliminar na Joanna Briggs Institute (JBI) Database of Systematic Reviews and Implementation Reports, Cochrane Database of Systematic Reviews, na Cumulative Index to Nursing and Allied Health Literature (CINAHL) (via EBSCO) e na Medical Literature Analysis and Retrieval System Online (MEDLINE), via PubMed, não foram encontradas qualquer tipo de revisões da literatura publicadas neste âmbito temático ${ }^{(13)}$.

Esta scoping review pretende contribuir para a uniformização na atuação no âmbito da Saúde Escolar, com base na referência num peso máximo de mochila recomendado nos estudos científicos, "onde a disciplina da Enfermagem, nomeadamente o enfermeiro especialista em Reabilitação poderá desenvolver ações para a prevenção de doenças e para o fortalecimento dos fatores de proteção" (13; 50), através da atuação concertada na implementação de Programas de Educação Postural (PEP), devidamente sustentados.

Esta revisão da literatura tem como objetivos: (a) mapear a evidência em relação ao peso máximo da mochila recomendado para crianças dos 6 aos 12 anos em contexto escolar; (b) identificar recomendações relacionadas com o tema; (c) identificar áreas de pesquisa e futuras investigações.

\section{MÉTODOS}

De forma a aprofundar os conhecimentos relativos à presente temática, elaborou-se um protocolo de scoping review ${ }^{(13)}$, tendo sido formulada a questão de partida: qual o peso máximo da mochila recomendado para crianças, dos 6 aos 12 anos, em contexto escolar?

Esta scoping review adotou a metodologia sugerida pelo Joanna Briggs Institute (JBI), descrita pelo The JBI Reviewers' Manual ${ }^{(14)}$. Com este método pretendeu-se fornecer um mapa do alcance das evidências disponíveis, quando alguma questão específica ainda não está clara, como é o caso ${ }^{(14)}$.

Foram definidos e aplicados critérios de seleção segundo a metodologia PCC (P- População, C - Conceito, C - Contexto):

- População (P): crianças dos 6 aos 12 anos, independentemente do género;

- Conceito (C): peso máximo da mochila recomendado para crianças;

- Contexto (C): escolas do ensino básico (EB) $\left(1^{\circ}\right.$ e $2^{\circ}$ ciclos de estudos), independentemente da localização geográfica e do sistema educacional.

Entre os meses de fevereiro e julho de 2018 foram analisados os artigos apresentados em texto completo (full text) e que o idioma contemplasse a língua portuguesa, inglesa e espanhola. Todas as referências citadas pelos artigos incluídos foram tidas em consideração.

Incluíram-se estudos primários que abrangessem a temática estudada, em bases de dados, diretrizes internacionais publicadas e literatura cinzenta. Nos estudos com crianças que apresentassem um intervalo etário díspar estabeleceu-se como critério de inclusão os estudos em que a média de idades dos participantes fosse inferior a 13 anos. Como critérios de exclusão não foram incluídos artigos de opinião e notícias.

A estratégia de pesquisa dividiu-se em três etapas: primeiro, realizou-se uma pesquisa inicial nas bases de dados Cumulative Index to Nursing and Allied Health Literature (CINAHL), Medical Literature Analysis and Retrieval System Online (MEDLINE), Mediclatina, Sportdiscus, Academic Searchcomplete, via EBSCO Host Online Research Databases, onde foram analisadas as palavras contidas no título, resumo e palavras-chave.

Numa segunda pesquisa, foram utilizadas as palavraschave e/ou descritores devidamente selecionados: schoolbag; backpack*; load*; safe weight; load carriage; child*; school*, que deu origem à frase booleana schoolbag OR backpack* AND load* AND safe 
weight OR load carriage AND child* AND school* NOT adult* NOT adolescen*. Esta fase incluíu uma busca nas bases de dados Institute for Scientific Information (ISI) Web of Science; CINAHL, MEDLINE, Mediclatina, Sportdiscus, Academic Searchcomplete; National Center for Biotechnology Information (NCBI)/PUBMED. Foi realizada também pesquisa no Repositório Científico de Acesso Aberto de Portugal (RCAAP).

Por último, foi criada uma lista de todas as publicações encontradas.

Os dados foram extraídos dos artigos incluídos, por dois revisores independentes, usando um instrumento tipo tabela (tabela 1), desenvolvido especificamente para esta revisão, tendo em conta detalhes específicos sobre a população, o conceito, o contexto e os métodos de pesquisa com relevância para a questão e para os objetivos enunciados desta scoping review, conforme indicado pela metodologia desenvolvida pelo JBI.

\begin{tabular}{l|l}
\hline \multicolumn{2}{c}{ ARTIGO ANALISADO } \\
\hline Revisor: & Data de extração: \\
\hline Base de dados & Título \\
\hline Cód. Artigo & \\
\hline Autores & \\
\hline Ano & \\
\hline País & \\
\hline Especialidade & \\
\hline População/Amostra & \\
\hline Metodologia & \\
\hline Resultados/Detalhes & \\
Peso mochila recomendado & \\
\hline Sugestões/Recomendações/ \\
Comentários do revisor
\end{tabular}

Tabela 1 - Instrumento de extração de dados dos estudos analisados. Porto, Portugal, 2018

Os revisores confrontaram os achados das pesquisas realizadas nas diferentes bases de dados, verificaram as diferenças e resolveram as discordâncias nas descobertas, culminando nos resultados desta scoping review.

\section{RESULTADOS}

Foram identificadas 353 publicações nas diferentes bases de dados. Salienta-se que não foi encontrada nenhuma bibliografia cinzenta inerente ao tema, no RCAAP.

O processo de extração dos artigos está esquematizado, em forma de diagrama Prisma, na figura 1.

No final da última etapa foram incluídos 28 estudos, para integrar a amostra, dos quais seis surgiram a partir das referências citadas (tabela 2$)^{1}$.

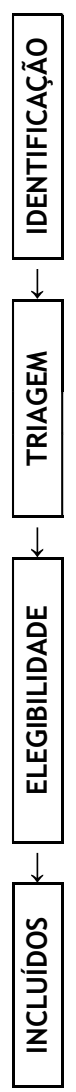

ARTIGOS IDENTIFICADOS NAS BASES DE DADOS $(n=353)$

ARTIGOS IDENTIFICADOS PELAS REFERÊNCIAS $(n=30)$

\section{$\downarrow$ \\ ARTIGOS DUPLICADOS REMOVIDOS $(n=62)$}

ARTIGOS ANALISADOS $(\mathrm{n}=321)$

$\downarrow$

$\downarrow$

ARTIGOS COMPLETOS ANALISADOS $(n=90)$

ARTIGOS COMPLETOS EXCLUÍDOS $(n=62)$

\section{ESTUDOS INCLUÍDOS NA REVISÃO $(n=28)$}

Figura 1 - Diagrama PRISMA do processo de seleção de estudos. Porto, Portugal, 2018

Na tabela 3 apresentam-se os objetivos de cada um dos 28 estudos incluídos.

A maioria dos estudos aborda a relação do peso da mochila escolar com a postura corporal e/ou com a dor, o efeito da mochila nas forças de reação/pressão plantar e os parâmetros da marcha e o impacto do peso da mochila na função ventilatória.

Relativamente aos anos de publicação dos estudos incluídos nesta scoping review, tal como se pode verificar no gráfico 1 , vinte e dois dos estudos foram publicados a partir do ano de $2013^{\text {(R2-R22) }}$ e seis antes deste ano ${ }^{(\mathrm{R} 23-\mathrm{R} 28)}$.

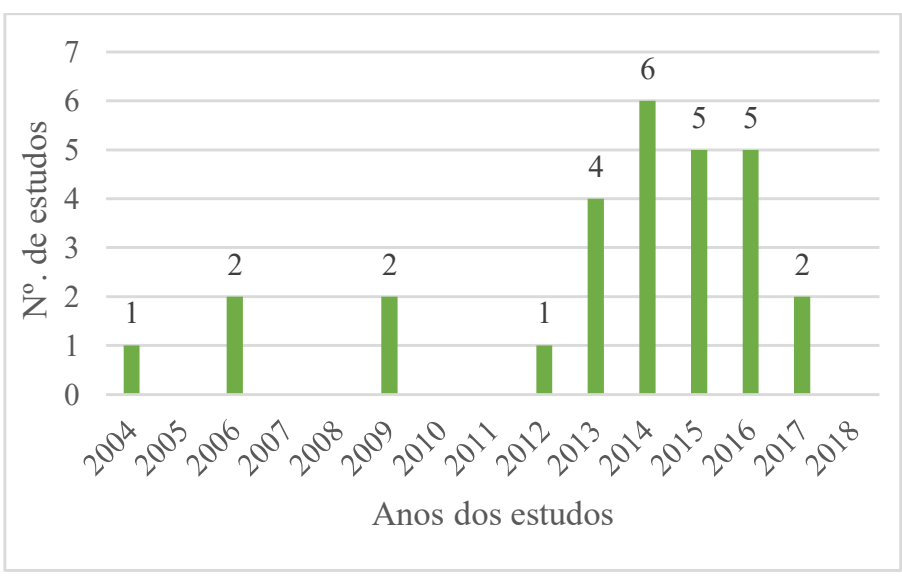

Gráfico 1 - Distribuição do ano de publicação de estudos. Porto, Portugal, 2018

\footnotetext{
${ }^{1}$ Os 28 estudos incluídos serão identificados doravante com a designação " $R$, seguido da numeração 1 a 28 (tabela 2).
} 
R1 Mosaad DM, Abdel-Aziem AA. Backpack carriage effect on head posture and ground reaction forces in school children. Work. 2015; 52(1): 203-209. DOI: 10.3233/WOR-152043

R2 Khallaf ME, Fayed EE, Ashammary RA. The effect of schoolbag weight on cervical posture in schoolchildren. Turk J Phys Med Rehab. 2016; 1(62): 16-21. DOI: 10.5606/tftrd.2016.12754.

R3 Silva D, Soares H, Rodrigues MA. Schoolbag weight and the postural and psychophysical changes in young Students: a Pilot Study. Occupational Safety and Hygiene IV. 2016: 453-457 DOI: http://dx.doi.org/10.1016/j.ergon.2015.09.010

R4 Dockrell S, Blake C, Simm C. Guidelines for schoolbag carriage: an appraisal of safe load limits for schoolbag weight and duration of carriage. Work. 2016; 53: 679-688. DOI: 10.3233/WOR-162260.

R5 Drzał-Grabiec J, et al. Effect of asymmetrical backpack load on spinal curvature in school children. Work. 2014; 51(2): 1-6. DOI: $10.3233 /$ WOR-141981

R6 Al-Katheri AE. Impact of backpack load on ventilatory function among 9-12 year old Saudi girls. Saudi Medical Journal. 2013; 34(12): 1255-1261. DOI: 10.1080/00140139.2014.997803

R7 Adeyemi AJ, Rohani JM, Rani MRA. Interaction of body mass índex and age in muscular activities among backpack carrying male schoolchildren. Journal of Prevention Assessment \& Rehabilitation. 2014; 52(3): 677-686. DOI: 10.3233/WOR-152102

R8 Kabilmiharbi N, Santhirasegaram TA. Study on relationship between carrying schoolbags and the prevalence of neck and back pain among 7 - 9 year old students. MATEC Web of Conferences. 2017; 87. DOI: 10.1051/matecconf/20178702013

R9 Bahiraei S, Jafarian M, Mohammad E. The effects of backpack weight on the static and dynamic balances of female students. Annals of Applied Sport Science. 2015; 3(3): 57-66.

R10 Mo SW, et al. Effect of backpack load on the head, cervical spine and shoulder postures in children during gait termination. Ergonomics. 2013; 56(12): 1908-1916. DOI: 10.1080/00140139.2013.851281

R11 Dianat I, et al. The use of schoolbags and musculoskeletal symptoms among primary school children: are the recommended weight limits adequate? Ergonomics. 2014; 56(1): 79-89. DOI: 10.1080/00140139.2012.729612

R12 Malik M, Vinay D, Kumkum P. Assessment of change in cervical and shoulder posture due to carriage of different weight of backpack. 2017; 9(2): 1266-1275. DOI: 10.31018/jans.v9i2.1353

R13 Adeyemi AJ, Rohani JM, Rani MR. Backpack-back pain complexity and the need for multifactorial safe weight recommendation. Applied Ergonomics. 2016: 1-10. DOI: 10.1016/j.apergo.2016.04.009

R14 Song Q, et al. Effects of backpack weight on posture, gait patterns and ground reaction forces of male children with obesity during stair descent. Research in Sports Medicine: An International Journal. 2014; 22(2): 172-184. DOI: $10.1080 / 15438627.2014 .881823$

R15 Zhou N, et al. Effects of daily used bags on biomechanics characteristic of elementary. 2016; 16(4): 265-274. DOI: $10.24264 /$ lfj.16.4.2

R16 Usman G, Agha S, Ameen F. Effects of heavy bags, plus desks and postural variations association with lower back pain in school going children. Gomal University Journal of Research. 2014; 30(1): 76-83.

R17 Dockrell S, Simms C, Blake C. Guidelines on schoolbag use: messaging to inform the stakeholders. Work. 2016; 54: 489-492. DOI: $10.3233 /$ WOR-162260

R18 Vieira AC, Ribeiro F. Impact of backpack type on respiratory muscle strength and lung function in children. Ergonomics. 2015; 58(6): 1005-1011. DOI: 10.1080/00140139.2014.997803

R19 Walicka-Cuprys K, et al. Influence of the weight of a school backpack on spinal curvature in the sagittal plane of seven-yearold children. BioMed Research International. 2015; 1-6. DOI: 10.1155/2015/817913

R20 Zhou N, et al. Investigation of the effect of varied schoolbag weight on the health of primary. Pielarie Incaltaminte. 2015; 15(3): 159-168. DOI: $10.24264 /$ lfj.15.3.3

R21 Terpstra R. September 17 is National School Backpack. Curriculum Review. 2014; 54(1)

R22 Kistner F, et al. Postural compensations and subjective complaints due to backpack loads and wear time in schoolchildren. Pediatric Physical Therapy. 2013; 25(1): 15-24. DOI: 10.1097/PEP.0b013e31827ab2f7

R23 Shasmin, H. N., et al. A Preliminary Study of Acceptable Load Carriage for Primary School Children. Biomed. 2006; 15: 171174. DOI: $10.1007 / 978-3-540-68017-8 \_44$

R24 Bauer, DH, Freivalds, A. Backpack load limit recommendation for middle school students based on physiological and psychophysical measurements. Work. 2008; 32: 339-350. DOI: 10.3233/WOR-2009-0832

R25 Dockrell S, Simms C, Blake C. Schoolbag weight limit: can it be defined? Journal of School Health. 2013; 83(5): 368-377. DOI: $10.1111 /$ josh.12040.

R26 Brackley HM, Stevenson JM. Are children's backpack weight limits enough? A critical review of the relevant literature. Spine. 2004; 29(19): 2184-2190. DOI: 10.1097/01.brs.0000141183.20124.a9

R27 Lindstrom-Hazel D. The backpack problem is evident but the solution is less obvious. Work. 2009; 32: 329-338. DOI: 10.3233/WOR-2009-0831.

R28 Razali R, et al. Accetable load carriage for primary school girls. European Journal of Scientific Research. 2006; 15(3): 396 403. 


\section{OBJETIVOS}

R1 - Avaliar o efeito do transporte da mochila nos ângulos do pescoço e forças de reação ao solo em crianças.

R2 - Relacionar o efeito de várias cargas da mochila escolar sobre a postura cervical durante a postura estática e após a caminhada.

R3 - Analisar a aplicabilidade de um novo procedimento experimental para determinar mudanças biomecânicas e psicofísicas em jovens estudantes, durante o transporte da mochila escolar em um e dois ombros.

R4 - Analisar a existência de desconforto musculoesquelético com base na percentagem de peso corporal, peso da mochila escolar e duração do transporte.

R5 - Avaliar os parâmetros da postura corporal no plano sagital para uma carga de mochila assimétrica igual a 10\% da massa corporal de uma criança.

R6 - Explorar a carga de mochila sob a forma de percentil do peso corporal e seu impacto na função ventilatória.

R7 - Analisar a interação entre o índice de massa corporal e a idade nas atividades musculares, a altura da mochila e o nível percebido de dor, decorrente do peso diferente das mochilas escolares.

R8 - Relacionar o modo de carregar a mochila escolar e a prevalência de dor no pescoço e nas costas nos estudantes.

R9 - Analisar os efeitos da mochila escolar no equilíbrio de estudantes do género feminino.

R10 - Quantificar os efeitos de carregar diferentes cargas de mochila na postura durante a marcha.

R11 - Analisar a relação entre o uso de mochilas escolares e a prevalência de sintomas musculoesqueléticos.

R12 - Medir a variação percentual no ângulo de postura cervical e ombros dos alunos devido ao transporte de diferentes pesos de mochila.

R13 - Analisar a dor nas costas relacionada com a mochila escolar e outros fatores de risco relacionados.

R14 - Analisar os efeitos do peso da mochila na postura, no padrão de marcha e nas forças de reação ao solo nas crianças com obesidade.

R15 - Avaliar os efeitos das mochilas escolares usadas diariamente nas características biomecânicas.

R16 - Relacionar o uso das mochilas escolares pesadas e das mesas escolares nas variações posturais e lombalgia em crianças.

R17 - Fornecer linhas orientadoras para o transporte de mochilas escolares, como base de conhecimento para as partes interessadas, incluindo usuários (crianças), pais, fabricantes de mochilas escolares e outras partes interessadas, como organizações de professores, profissionais de saúde e políticos.

R18 - Analisar a influência do tipo mochila na função pulmonar e força muscular respiratória em crianças.

R19 - Relacionar o peso da mochila escolar, com o peso corporal da criança e a sua postura corporal

R20 - Explorar os efeitos de pesos diferentes de mochilas escolares sobre a biomecânica dos alunos do ensino fundamental.

R21 - Informar sobre o dia nacional da consciencialização sobre a mochila escolar.

R22 - Analisar os efeitos do transporte de mochilas pesadas até $20 \%$ do peso corporal sobre a postura e queixas álgicas de crianças do ensino fundamental.

R23 - Analisar as mudanças nas forças de reação ao solo e inclinação do tronco entre os alunos do ensino fundamental ao carregar mochilas escolares pesadas.

R24 - Avaliar a postura, a frequência cardíaca, o esforço percebido e a perceção da dor para encontrar um limite de carga de mochila aceitável para estudantes do ensino médio.

R25 - Rever a literatura sobre mochilas escolares com ênfase especial nas diretrizes de limite de carga e dor musculoesquelética.

R26 - Rever a literatura sobre a epidemiologia, fisiologia e biomecânica que contribui para estabelecer um limite de peso para as mochilas escolares das crianças.

R27 - Rever a literatura sobre o risco de dores nas costas nas crianças em idade escolar associado ao transporte de mochilas escolares.

R28 - Analisar como o aumento da carga da mochila afeta a marcha e a postura em crianças do ensino fundamental. 
Quinze estudos foram realizados no continente asiático (R2, R6-R16, R20, R23, R28), sete na Europa (R3-R5, R17-R19 R25), cinco na América do Norte (R21-R22, R24, R26-R27) e um estudo é proveniente do continente africano ${ }^{\mathrm{R} 1}$

No que diz respeito à especialidade/área de intervenção dos autores dos estudos a maioria dos artigos foram realizados no âmbito da Engenharia (R3, R7R8, R13, R15, R20, R23-R24, R28), da Fisioterapia (R1-R2, R4, R6, R17, R19, R22, R25) e na área das Ciências do Desporto (R5, R9-R10, R14, R26). Os restantes distribuem-se por especialidades como a Terapia Ocupacional (R21, R27), Saúde Ocupacional (R11), Gestão de Recursos Familiares ${ }^{(\mathrm{R} 12)}$, Saúde Comunitária (R16) e Ciências da Saúde (R18).

Em relação às amostras utilizadas, nos diferentes estudos incluídos, há uma oscilação entre 5 e 500 crianças participantes.

Três estudos foram realizados apenas com crianças do género feminino ${ }^{(R 6, R 9, R 28)}$ e quatro do género masculino $(\mathrm{R} 3, \mathrm{R} 7, \mathrm{R} 10, \mathrm{R} 23)$. Todos os restantes foram realizados com crianças de ambos os géneros.

As idades incluídas nos estudos desta revisão da literatura vão desde os 6 até aos 15 anos, mas a média de idades dos participantes foi sempre inferior a 13 anos, de forma a dar resposta aos critérios PCC definidos.

Quanto à metodologia de pesquisa constatou-se que doze estudos são de caráter experimental (R3, R6-R7, R10, $\mathrm{R} 12, \mathrm{R} 14-\mathrm{R} 15, \mathrm{R} 20, \mathrm{R} 22-\mathrm{R} 24, \mathrm{R} 28)$, sete descritivo-correlacionais (R2, R4-R5, R11, R16, R18-R19) e três são revisões da literatura (R25-R27). Os outros estudos utilizaram o método de pesquisa descritivo ${ }^{(R 8-R 9)}$, multicasos ${ }^{(R 1)}$ e misto ${ }^{(R 13)}$. Dois dos artigos selecionados são diretrizes/orientações quanto ao peso recomendado da mochila escolar (R17, R21).

Em contexto escolar, no que diz respeito às recomendações acerca do peso máximo da mochila, em relação ao peso corporal das crianças, dos 6 aos 12 anos, dez estudos recomendam o valor de $10 \%$ (R3-R5, R8, R10-R11, R19, R22, R24, R28), sete indicam que as mochilas só devem pesar até 15\% (R14-R16, R18, R20-R21, R23), dois estudos sugerem valores inferiores a 7,5\% (R1, R6) e cinco recomendam intervalos de peso entre os 5 e os $20 \%$ (R2, R9, R12, R25-R26). Quatro estudos afirmam não ser prudente recomendar percentagem de peso da mochila em relação ao peso corporal da criança (R7, R13, R17, R27).

De acordo com as investigações integradas nesta revisão de literatura, as consequências de carregar mochilas pesadas podem acarretar nas crianças complicações a vários níveis, tais como: dor no pescoço, ombros e costas (R11-R13, R16, R21-R22, R25-R26, R28), alterações posturais (a nível das curvaturas da coluna vertebral) (R1-R3, R5, R10, R12, R14-R16, R19, R22-R24), alteração do equilíbrio e condicionamento de movimentos ${ }^{(R 9)}$, diminuição da função ventilatória (R6, R18) e alterações na pressão plantar e marcha ${ }^{(\mathrm{R} 20, \mathrm{R} 23)}$.

As recomendações para colmatar as consequências do peso excessivo da mochila, podem ser identificadas na tabela 4.

Como áreas de pesquisa futura, sete estudos indicam que será fundamental avaliar longitudinalmente e no ambiente escolar natural as consequências do peso excessivo da mochila, a nível das alterações das curvaturas da coluna vertebral (R1-R3, R10, R27) e ao nível da dor nas costas (R27) e das queixas psicossomáticas da criança (R27). Cinco artigos sugerem, também, que se verifique a relação entre o transporte da mochila e outros fatores de risco para o desenvolvimento de LME nas crianças (R4, R8, R25-R26). Outros artigos propõem o estudo da perceção de peso da mochila pela criança (peso percebido) ${ }^{(\text {R27); }}$ bem como a avaliação da aptidão física no transporte da mesma (R26). Um artigo menciona, também, a importância do estudo sobre o design das mochilas e as adaptações necessárias para o seu transporte ${ }^{(\mathrm{R} 26)}$.

\section{RECOMENDAÇÕES}

R4, R8, Melhoramento do design das mochilas R12, R26 R4, R5, Postural, por professores, fisioterapeutas ou R24, R25 enfermeiros, como parte do currículo escolar, incluindo os alunos, pais e professores, sem descurar as especificidades de cada contexto/individuais

R2, R5 Rastreio regular da postura corporal e do peso da mochila

R12, R19 Política de redução da carga/número de livros/gestão de livros escola $\leftrightarrow$ casa

R4, R18 Uso de mochila com duas alças

R21 Realização de testes visuais das zonas de pressão relacionadas com a carga excessiva da mochila

R25 Aumento do número de cacifos escolares

R14 Diminuição da percentagem de peso da mochila recomendado para crianças obesas

Tabela 4-Recomendações para colmatar as consequências do peso excessivo da mochila dos artigos incluídos. Porto, Portugal, 2018

\section{DISCUSSÃO}

Com esta scoping review procurou-se não só perceber o que é que os artigos incluídos sugerem em relação à percentagem de peso máximo da mochila recomendado, como evidenciar outros aspetos intrinsecamente relacionados com este tema, como as consequências de carregar mochilas pesadas e, algumas recomendações para prevenir/colmatar essa situação. Por este motivo, perceber os aspetos que interferem na saúde das crianças, será importante para que os profissionais de saúde possam implementar uma prática baseada na evidência.

Perante a análise dos resultados, os achados não foram uniformes, subdividindo-se em duas categorias: os que recomendam percentagem de peso máximo da mochila escolar (que oscila entre os $5 \%$ e os $20 \%$ ) e os que não recomendam qualquer tipo de percentagem, principalmente dado o aumento do número de crianças obesas, pois o cálculo baseado na percentagem de peso corporal, poderá não ser uma abordagem apropriada. 
De realçar que o valor de $10 \%$ foi o que obteve maior consenso.

Deste modo, em relação à questão colocada inicialmente, conclui-se que a literatura defende que o conteúdo da mochila escolar não deve exceder os $10 \%$ do peso corporal da criança em contexto escolar. Porém, não deverá ser negligenciado que poderá não ser prudente recomendar uma percentagem de peso fixa. Por um lado, o peso da mochila escolar expresso em percentagem, poderá não ser o único fator associado aos sintomas musculoesqueléticos nas crianças do EB, dado, por exemplo, o aumento atual dos níveis de obesidade entre os estudantes (R7) . Por outro, em relação aos limites de peso máximo da mochila recomendados que, geralmente, são propostos para as crianças, embora cientificamente não consensuais, são largamente excedidos na vida quotidiana ${ }^{(15)}$.

Deste modo, as consequências mais prevalentes do transporte de mochilas pesadas ocorrem a nível musculoesquelético, mas outros sistemas anátomofisiológicos da criança também poderão estar comprometidos, devido ao transporte de mochilas com peso em excesso.

Esta revisão mostrou-se abrangente em termos de localização dos estudos incluídos. Contudo, destaca-se que a maioria dos estudos são oriundos da Ásia e apenas dois, dos estudos europeus, são portugueses. Mais nenhum estudo é originário de um país de língua portuguesa/espanhola.

A variabilidade de estudos incluídos vem confirmar a complexidade e a abrangência de fatores que caracterizam esta questão e como constatado, esta pesquisa abarcou estudos dos últimos 14 anos, o que lhe confere uma grande amplitude.

Os estudos incluídos nesta scoping review não são uniformes quanto à constituição da amostra em número, em género e em idade e quanto à metodologia adotada. Como tal, alguns destes estudos não permitem generalizar os resultados, o que se constitui como uma limitação dos mesmos.

Será importante referir que a interpretação dos resultados desta scoping review deverá ser efetuada de forma prudente, dadas as limitações identificadas nos estudos incluídos. Também, não ter sido incluída literatura cinzenta internacional poderá ter limitado alguma da pesquisa científica realizada neste âmbito.

De realçar que, a nível mundial, nenhum estudo sobre este tema, incluído nesta revisão, foi realizado pela área da Enfermagem. Porém, esta disciplina ao se interessar e intervir nos fenómenos que afetam a Saúde Escolar está intrinsecamente envolvida com esta problemática. Também, a carência de estudos, tanto em formato de artigos científicos, como de bibliografia cinzenta, em Portugal, sobre este tema, reforça a importância da Enfermagem de Reabilitação investir nesta área a nível investigacional.

Assim, um conhecimento, com contributos multidisciplinares, dos fatores que condicionam a saúde da criança, permite ao enfermeiro, dotar a sua intervenção de maior rigor, no sentido de minimizar os efeitos da sobrecarga inerentes ao transporte da mochila escolar, nomeadamente, na adoção de práticas seguras $^{(15)}$.

Tal como sugere a Organização Mundial da Saúde grande parte da morbilidade nas crianças e jovens é evitável, sendo que medidas de baixo custo demonstram ser eficazes na prevenção do abuso de substâncias, lesões e transtornos mentais, entre outros $^{(16)}$. Neste caso específico, há uma ampla variabilidade de medidas para colmatar as consequências do peso excessivo da mochila, que se consideram como medidas de baixo valor económico, e, como tal, passíveis de ser facilmente implementadas.

A nível laboral, existe legislação que delimita o peso máximo, nas tarefas que impliquem a movimentação manual de cargas, para os adultos. Contudo, verifica-se que ainda não foram desenvolvidos limites e diretrizes para aplicação em crianças em idade escolar, no que diz respeito a este assunto.

Embora a importância económica deste problema possa ser pequeno a curto prazo, as taxas de dor lombar nas crianças aproximam-se das observadas em adultos, o que poderá trazer consequências a médio e a longo prazo $^{(10)}$.

Até que um consenso sobre um limite de carga universal seja acordado e a publicação de diretrizes e/ou de legislação que todos possam entender seja difundida, estudantes, pais, professores, profissionais de saúde e todos os outros envolvidos deverão tomar consciência do problema inerente ao transporte de mochilas pesadas, promovendo as medidas necessárias para ajudar a prevenir lesões nas crianças.

\section{CONCLUSÃO}

Com a síntese dos resultados de pesquisas com relevância mundial, através da metodologia scoping review, foi possível perceber qual a percentagem de peso máximo da mochila recomendado para crianças (612 anos) em contexto escolar, no sentido de contribuir para a incorporação do conhecimento científico na prática profissional da Enfermagem.

Com esta revisão da literatura, permitiu-se a transferência futura de evidências ao destacar-se aspetos inerentes ao peso máximo da mochila recomendado para crianças, dos 6 aos 12 anos em contexto escolar, conforme a literatura. O valor de $10 \%$ foi o que obteve maior consenso. Assim, para fazer a diferença na promoção da saúde escolar, é imprescindível vincular o conhecimento oriundo de pesquisas, na prática clínica.

Posto isto, as deficiências na literatura atual devem ser abordadas, para que orientações baseadas em evidências possam ser fornecidas aos alunos, pais, professores e restantes partes interessadas. Sugere-se, assim, que uma diretriz geral possa ser mais apropriada do que uma diretriz de limite de carga única, ou seja, em vez de se tentar estabelecer um único limite de carga para todas as crianças, o foco da pesquisa deverá incidir nos fatores que afetam o transporte da mochila escolar. 
Estas sugestões deixam em aberto um leque elevado de áreas de pesquisa futuras, que poderão contribuir para um maior conhecimento e sustentação das consequências do transporte da mochila escolar em crianças dos 6 aos 12 anos.

Pelo que foi supramencionado, e devido a alguns aspetos que carecem ainda de consenso, sugere-se a realização de uma revisão da literatura, para a identificação conjugada dos fatores de risco (ex. design da mochila; características individuais; peso percebido; fatores psicossomáticos; entre outros) que contribuem para as LME e dor nas costas nas crianças em idade escolar.

Pela gravidade deste problema, toda a CE deverá ser educada na área da Ergonomia. Também, o rastreio regular da postura da criança e do peso da mochila, ajustado aos diferentes contextos e a implementação de Programas de Educação Postural, como parte do currículo académico, por enfermeiros de reabilitação, surgem como recomendações-chave na redução do conteúdo da mochila e na gestão do transporte dos livros escola $\leftrightarrow$ casa.

Muitas das soluções para o problema implícito ao peso das mochilas escolares são encontradas localmente em cada CE. Os projetos realizados por estudantes e/ou professores em colaboração com os pais e profissionais de saúde, que se concentrem em cada individuo/turma/escola/agrupamento escolar deverão ser valorizados. Também, por exemplo, a provisão de armários escolares ou o melhoramento da acessibilidade aos mesmos poderá ser uma solução.

As estratégias contínuas baseadas em cada CE deverão visar, igualmente, a divulgação de informações sobre o que é conhecido, que incluam a consciencialização sobre o peso e tipologia ideal das mochilas e os recursos escolares específicos, através de vários meios de comunicação, tais como campanhas publicitárias, palestras/participação em reuniões de pais, entre outras, usando formas de comunicação já existentes ou novas.

Para tal, é prioritária a necessidade de aumentar o número de enfermeiros nas escolas, para que exista uma intervenção sustentada em consonância com as necessidades de cada $\mathrm{CE}$, numa lógica de atuação centrada no empoderamento dos intervenientes.

\section{REFERÊNCIAS BIBLIOGRÁFICAS}

1. Mosaad DM, Abdel-Aziem AA. Backpack carriage effect on head posture and ground reaction forces in school children. Work. 2015; 52(1): 203-209. DOI: 10.3233/WOR-152043.

2. Khallaf ME, Fayed EE, Ashammary RA. The effect of schoolbag weight on cervical posture in schoolchildren. Turk J Phys Med Rehab. 2016; 1(62): 16-21. DOI: 10.5606/tftrd.2016.12754

3. Silva D, Soares H, Rodrigues MA. Schoolbag weight and the postural and psychophysical changes in young students: a pilot study. Occupational Safety and Hygiene IV, 2016: 453-457. DOI: 978-1-13802942-2.

4. Pinho MERC, et al. Lesões musculoesqueléticas relacionadas com as atividades desportivas em crianças e adolescentes: uma revisão das questões emergentes. Motricidade. 2013; 9(1): 31-49. DOI: 10.6063/motricidade.9(1).2461

5. Carnide MF. Ergonomia Escolar: Recomendações. Programa Nacional de Saúde Escolar: Programa Nacional Contra as Doenças
Reumáticas. [Online] 2006. Disponível em: https://www.dgs.pt/ficheiros-de-upload-3/pncdr-recom-ergonomiaescolar_final-out06-pdf.aspx.

6. Moreira J, Cornelian, BR, Lopes, CPB. A importância do bom posicionamento postural em escolares - o papel do professor de educação física. Rev Uningá. [Online] 2013 Dec 10;16(3), 42-8. Disponivel em: http://revista.uninga.br/index.php/uningareviews/article/view/14 80.

7. Direção-Geral da Saúde. Programa Nacional de Saúde Escolar. Lisboa : Direcção-Geral da Saúde, 2015. 978-972-675-227.

8. Voll H, Klimt F. On Strain in Children Caused by Schoolbags. Offentliche Gesundheitswesen. 1977; 39: 369-378.

9. Dockrell S, Blake C, Simm C. Guidelines for schoolbag carriage: An appraisal of safe load limits for schoolbag weight and duration of carriage. Work. 2016; 53: 679-688. DOI: 10.3233/WOR-162260.

10. Drzat-Grabiec J, et al. Effect of asymmetrical backpack load on spinal curvature in school children. Work. 2014; 51(2): 1-6. DOI: 10.3233/WOR-141981

11. Al-Katheri AE. Impact of backpack load on ventilatory function among 9-12 year old Saudi girls. Saudi Medical Journal. 2013; 34(12): 1255-1261.

12. Adeyemi AJ, Rohani JM, Rani MRA. Interaction of body mass índex and age in muscular activities among backpack carrying male schoolchildren. Journal of Prevention Assessment \& Rehabilitation. 2014; 52(3): 677-686. DOI: 10.3233/WOR-152102.

13. Matos Maria, Barreiras Catarina, Festas Constança. Peso máximo da mochila recomendado para crianças (6-12 anos) em contexto escolar: um protocolo de scoping review. Rev Port Enferm Reabil [Online] 2019 Dec; 2(2): 49-52. Disponível em: https://www.aper.pt/ficheiros/revista/RPERv2n2.pdf DOl 10.33194/rper.2019.v2.n2.02.4575

14. Aromataris E, Munn Z (Editors). The Joanna Briggs Institute Reviewers' Manual. The Joanna Briggs Institute, 2017. Available from: https: / / reviewersmanual.joannabriggs.org/

15. Matos MJ. A intervenção do enfermeiro especialista em enfermagem de reabilitação na prevenção de lesões musculoesqueléticas na comunidade. [Relatório Mestrado em Enfermagem de Reabilitação]. Viana do Castelo: Instituto Politécnico de Viana do Castelo - Escola Superior de Saúde; 2017.

16. World Health Organization. Child and Adolescent Health. [Online] 2018. [Citação: 23 de March de 2018.] www.euro.who.int/en/healthtopics/Life-stages/child-and-adolescent-health. 